\title{
UNIVERSAL INFINITE CLIQUE-OMITTING GRAPHS
}

\author{
MIRNA DŽAMONJA \\ Dedicated to the memory of Professor Mahmut Bajraktarević
}

\begin{abstract}
The main result of the paper is that when $\kappa$ is a cardinal of cofinality $\omega$ and $\lambda \geq \kappa$, the class of graphs of size $\lambda$ omitting cliques of size $\kappa$ has no universal element under graph homomorphisms (or the weak and strong embeddings). This theorem only requires ZF.
\end{abstract}

\section{INTRODUCTION}

In graph theory one considers various ways in which graphs can embed into each other. The strongest one is a strong isomorphism, which is an injective function preserving both edges and non-edges, so this is an isomorphic embedding in the sense of model theory. If only the edges are preserved, we are in the situation of a weak isomorphism. The weakest notion is that of homomorphism, an embedding which is not necessarily injective, but it preserves edges. Working in the class of simple graphs, we observe that homomorphisms are injective on cliques. If we are given a class of graphs, a universal element of the class is one in which all the other elements of the class embed, under the notion of the embedding specified in the context. Model theorists studying infinite graphs most often work with the notion of strong embedding, but graph homomorphisms are a very well studied notion in the class of finite graphs, among other reasons because they are connected with the notion of a graphon, an uncountable combinatorial limit of finite graphs (see Lovasz's book [5] for more information).

Let $\kappa \leq \lambda$ be cardinals. We shall work with graphs of size $\lambda$ with no cliques of size $\kappa$. The class of such graphs will be denoted by $\mathcal{K}_{\lambda, \kappa}$ and we shall be looking for a universal under graph homomorphisms element in that

2010 Mathematics Subject Classification. 03E05, 05C63.

Key words and phrases. Universal graph, omitting infinite cliques, countable cofinality. The author thanks l'Institut d'Histoire et de Philosophie des Sciences et des Techniques, Université Paris 1, where she is an Associate Member.

Copyright (c) 2016 by ANUBIH. 
class. The existence of such an element depends on the choice of $\lambda$ and $\kappa$, for example for $\lambda=\aleph_{0}$ and $\kappa$ finite the existence follows either vacuously or by the Fraïssé construction in model theory. Things get more complicated when $\kappa$ is infinite. Komjáth and Shelah resolve the problem in [4] but only under the additional assumption of GCH (generalised continuum hypothesis):

Theorem 1 (Komjáth-Shelah). If GCH holds, then for infinite $\kappa$ there is a universal element in $\mathcal{K}_{\lambda, \kappa}$ under strong (weak) embeddings iff the cofinality of $\kappa$ is strictly larger than that of $\lambda$.

This theorem in particular implies that under the assumption of $\mathrm{GCH}$, when $\kappa$ is a cardinal of countable cofinality, there is no universal element in any $\mathcal{K}_{\lambda, \kappa}$ under strong or weak embeddings. The main result of this paper is that neither the assumption of GCH nor of strong or weak embeddings is necessary, moreover, even the axiom of choice is unnecessary, as we exhibit a proof of the corresponding negative universality result under homomorphic embeddings and entirely within ZF (Theorem 3). We note, however, that our proof is very specific to the countable cofinality. In the positive direction, [4] gives a direct proof, for any infinite $\kappa$, but it is also easy to obtain a proof by using the generalised Fraïssé constructions as introduced by Jónsson in [2], modifying slightly the proof of Theorem 2.11 there.

Compared to the set-theoretic universes where GCH holds, on the opposite end of the spectrum are the models that are far from GCH. Such models were studied by Kojman in [3]. He proved, using a technique of adjacency graphs, the following results:

Theorem 2 (Kojman). Let $\kappa$ be a cardinal $\geq 3$.

(1) Suppose that there is a cardinal $\theta$ such that $\operatorname{cf}\left(2^{\theta}\right) \leq \lambda<2^{\theta}$. Then $\mathcal{K}_{\lambda, \kappa}$ has no universal element under strong embeddings, and in fact the universality number of the class $\mathcal{K}_{\lambda, \kappa}$ is larger than $2^{\theta}$.

(2) If there is a cardinal $\theta$ such that $\theta<\lambda<2^{\theta}$ but $\operatorname{cf}\left(2^{\theta}\right)=\theta^{+}$, then $\mathcal{K}_{\lambda, \kappa}$ has no universal element under strong embeddings and moreover, the universality number of $\mathcal{K}_{\lambda, \kappa}$ is at least $2^{\theta^{+}}$.

\section{WhEN $\kappa$ HAS COUNTABLE COFINALiTy}

The cofinality $\operatorname{cf}(\kappa)$ of an infinite cardinal $\kappa$ is defined as the smallest size of an unbounded subset of $\kappa$ in the order given by considering $\kappa$ as an ordinal. An infinite cardinal number $\kappa$ is said to be regular if $\operatorname{cf}(\kappa)=\kappa$, otherwise $\kappa$ is said to be singular. It turns out that for $\kappa$ of countable cofinality, ZFC or in fact just ZF is sufficient to give the full conclusion on the possibility of the existence of a universal model in any $\mathcal{K}_{\lambda, \kappa}$, which is always negative. 
Theorem $3(\mathrm{ZF})$. Suppose that $\kappa$ is a cardinal of cofinality $\omega$ and $\lambda \geq \kappa$. Then $\mathcal{K}_{\lambda, \kappa}$ does not have a universal element under graph homomorphisms (or the weak and strong embeddings).

Proof. Given a graph $G \in K_{\lambda, \kappa}$, we shall construct a graph $H \in K_{\lambda, \kappa}$ such that $H$ does not admit a homomorphism into $G$.

Let $\left\langle\kappa_{n}: n<\omega\right\rangle$ be an increasing sequence of regular cardinals $\geq 2$ converging to $\kappa$ and let $\left\langle A_{n}: n<\omega\right\rangle$ be a sequence of pairwise disjoint sets, each disjoint from $G$ and such that $\left|A_{n}\right|=\kappa_{n}$ for each $n$. Now define the graph $H$ by letting its vertex set be that of $G$ together with $\bigcup_{n<\omega} A_{n}$. The edges of $H$ are those of $G$ together with edges making each $A_{n}$ a clique and edges connecting all the elements of any $A_{n}$ with all the elements of $G$ (but no edges are imposed between the elements of $A_{n}$ and $A_{m}$ for $n \neq m$ ). We can easily verify that the size of $H$ is $\lambda$ and that no $\kappa$-clique is added, hence $H$ still does not have any $\kappa$-cliques. Suppose for a contradiction that $f$ is a homomorphism from $H$ to $G$. For $1 \leq k<\omega$, let $f^{k}$ denote the composition of $f$ with itself $k$ times.

We claim that $\bigcup_{n<\omega} f^{n+1}\left(A_{n}\right)$ is a clique in $G$ of size $\kappa$, so a contradiction. To show this, it suffices to show that for each $n$ the set $\bigcup_{k \leq n} f^{k+1}\left(A_{k}\right)$ is a clique of size at least $\kappa_{n}$. Since graph homomorphisms are necessarily 1-1 on cliques ${ }^{1}$, it is clear that for every $k$ the set $f^{k+1}\left(A_{k}\right)$ has size $\kappa_{k}$, and it then suffices to show that $\bigcup_{k<n} f^{k+1}\left(A_{k}\right)$ is actually a clique. For $k=0$ this is easy as $A_{0}$ is a clique of size $\kappa_{0}$ and $f$ preserves edges. At the stage $n+1$ we have by the induction hypothesis that $\bigcup_{k<n} f^{k+1}\left(A_{k}\right)$ is a clique and by the properties of a homomorphism that $f^{n+2}\left(A_{n+1}\right)$ is a clique, so we need to show that every $x \in \bigcup_{k \leq n} f^{k+1}\left(A_{k}\right)$ is connected with every $y \in f^{n+2}\left(A_{n+1}\right)$. Let $k \leq n+1$ be such that $x \in f^{k+1}\left(A_{k}\right)$ and let $x^{\prime} \in A_{k}$ be such that $x=f^{k+1}\left(x^{\prime}\right)$. Let $y^{\prime} \in A_{n+1}$ be such that $y=f^{n+2}\left(y^{\prime}\right)$. Let $y^{\prime \prime}=f^{n-k+1}\left(y^{\prime}\right)$ and notice that since $n-k+1 \geq 1$, we have that $x^{\prime} E_{H} y^{\prime \prime}$. Applying $f^{k+1}$, we obtain that there is an edge in $G$ between $x=f^{k+1}\left(x^{\prime}\right)$ and $y=f^{k+1}\left(y^{\prime \prime}\right)$, as required.

It is easy to note that the above theorem generalizes to other classes of objects, such as partial orders of size $\lambda$ with no chains of size $\kappa$. Theorem 3 with the assumption that $\kappa=\lambda$ is a strong limit was proved by Galvin (unpublished) and the proof is given in [4].

2.1. In the absence of choice. In the system ZFC, every successor cardinal (so a cardinal $\kappa$ of the form $\kappa=\theta^{+}$for some $\theta$ ) is regular. However, the proof (due to Hausdorff) depends on the Axiom of Choice and without

\footnotetext{
${ }^{1}$ no vertex is edge-connected with itself
} 
it it is even possible that every uncountable cardinal is singular! To be exact, Gitik proved in [1] that from the consistency of ZFC and arbitrarily large strongly compact cardinals, it is possible to construct a model of ZF in which all cardinals have countable cofinality. As a consequence of Theorem 3 , in Gitik's model no $\mathcal{K}_{\lambda, \kappa}$ can have a universal element.

\section{REFERENCES}

[1] Moti Gitik, All uncountable cardinals can be singular, Israel J. Math., 35 (1-2) (1980), 61-88.

[2] Bjarni Jónsson, Universal relational systems, Math. Scand., 4 (1956), 193-208.

[3] Menachem Kojman, On universal graphs without cliques or without large bipartite graphs, Preprint, Research Showcase CMU, 1995.

[4] Peter Komjáth and Saharon Shelah, Universal graphs with no large cliques, J. Comb. Theory. Ser. B, 63 (1992), 125-135.

[5] László Lovász, Large networks and graph limits, volume 60 of American Mathematical Society Colloquium Publications, American Mathematical Society, Providence, RI, 2012.

(Received: August 1, 2016)

\author{
Mirna Džamonja \\ School of Mathematics \\ University of East Anglia \\ Norwich, NR4 7TJ \\ UK \\ M.Dzamonja@uea.ac.uk \\ http://www.mth.uea.ac.uk/people/md.html
}

